\title{
X-ray Fourier holography of free-flying nanoparticles
}

\author{
Femtosecond X-ray Fourier holography imaging of free-flying nanoparticle
}

Authors: Tais Gorkhover, Anatoli Ulmer, Ken Ferguson, Max Bucher, Filipe R. N. C. Maia, Johan Bielecki, Tomas Ekeberg, Max F. Hantke, Benedikt J. Daurer, Carl Nettelblad, Jakob Andreasson, Anton Barty, Petr Bruza, Sebastian Carron, Dirk Hasse, Jacek Krzywinski, Daniel S. D. Larsson, Andrew Morgan, Kerstin Mühlig, Kenta Okamoto, Alberto Pietrini, Daniela Rupp, Mario Sauppe, Gijs van der Schot, Marcin Siebert, Jonas A. Selberg, Martin Svenda, Michelle Swiggers, Nicusor Timneanu, Daniel Westphal, Garth Williams, Alessando Zani, Henry N. Chapman, Gyula Faigel, Thomas Möller, Janos Hajdu and Christoph Bostedt

Nature Photonics 12, 150 (2018)

\section{Recommended with a Commentary by J. B. Hastings, SLAC National Accelerator Laboratory}

Non-destructive imaging at the one to a few nanometer length scale has broad implications for science and technology. The obvious approach to imaging at the nanoscale is to extend Gabor holography [1] to a wavelength range corresponding to the desired resolution. This avoids the 'phase problem' that is inherent in trying to invert diffraction patterns from objects that in principle could have the desired resolution. An approach to resolving the phase problem was described in a short note by D. Sayre [2]. He presaged the development of coherent diffraction imaging postulating that by 'oversampling' one can retrieve the phase from a diffraction measurement that at the outset yields only the amplitudes of the complex structure factors needed to determine the electron density by Fourier methods. This idea was fleshed out by Fienup [3].

In parallel with the maturation of coherent diffraction imaging where resolutions approaching the $10 \mathrm{~nm}$ scale have been demonstrated, x-ray holography has been progressing with demonstration experiments. A recent example is the work of Eisebett and collaborators [4] where they looked at magnetic worm domains by creating a reference beam from a pinhole placed in the proximity of the sample held on an otherwise opaque support.

These parallel developments have a common goal. The imaging in three dimensions of isolated particles with resolutions below $1 \mathrm{~nm}$. The coherent imaging approach was the 'highlight' of the science case for hard x-ray free electron lasers where the long-term goal was diffraction imaging of isolated bio-molecules obviating the need for a crystal. This approach requires the acquisition of many two-dimensional diffraction patterns of sufficient resolution, assembling them into a 3-dimensional space and then through the iterative methods discussed 
above retrieve the real space molecular structure. While progress has been made, to date the best resolution on rather large virus particles is in the range 7.9-9 $\mathrm{nm}$ [5].

The challenge for the Fourier holographic approach, which has the advantage of directly encoding the phase in the scattering pattern, is the need for a reference beam. This is a significant issue recalling the experiment of Eisebett at al. where the reference beam is generated by a pinhole and the object is stationary as well. For holographic imaging of isolated objects they need to be 'injected' into the x-ray beam and at the same time as a reference beam created. Gorkhover and collaborators demonstrated a solution to this challenge: simultaneously inject a scattering source of essentially known shape and dimensions with the object of interest. In the demonstration experiment the reference beam comes from xenon clusters that have been studied previously by scattering methods and can be created with spherical shapes and known dimensions.

The authors also realized that although the relative positions of the reference scatterer and object are unknown this is not a problem. The approach yields high fidelity images of isolated nanosamples that are 'immune' to noise with the potential to reach sub-nanometer resolution electron densities using x-rays. From 1948 with the demonstration of in-line holography until today real-space images at the atomic scale have required crystals. One path, coherent diffraction imaging, to that goal for isolated nanoscale objects has made significant progress but has limitations. On the other hand, Fourier holography is just beginning with this first demonstration. Exploring the limits of this approach, utilizing the full 'power' of hard $\mathrm{x}$-tray free electron lasers, awaits. The advantages of the holographic approach are clear.

For true atomic scale resolution, we're not there yet but the potential of the holographic approach is clearly an advantage. In particular for imaging structural dynamics where the need for 'identical' particles to permit construction of a three dimensional intensity pattern in reciprocal space is unnecessary to solve the phase problem holography wins.

\section{References}

[1] D. Gabor et al., Nature 161, 777 (1948).

[2] D. Sayre, Acta Cryst. 5, 843 (1952).

[3] see for example, J. R. Fienup, Appl. Optics 21, 2758 (1982)

[4] S. Eisebitt et al., Nature 432, 885 (2004).

[5] Max Rose et al., IUCrJ 5, 727 (2018). 